
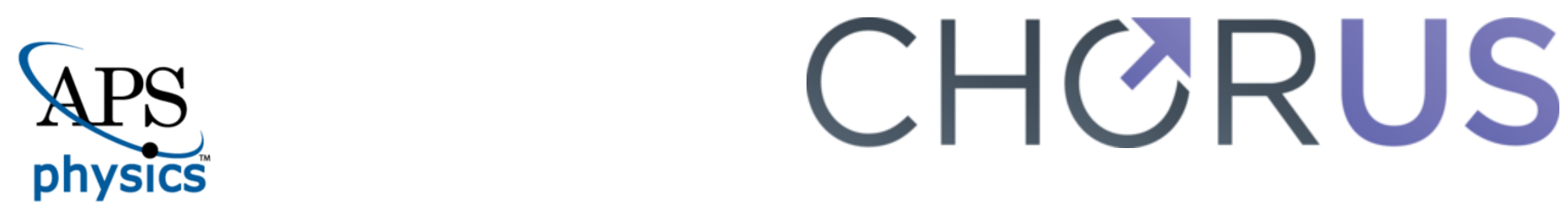

This is the accepted manuscript made available via CHORUS. The article has been published as:

\title{
Coulomb-repulsion-assisted double ionization from doubly excited states of argon
}

Qing Liao, Alexander H. Winney, Suk Kyoung Lee, Yun Fei Lin, Pradip Adhikari, and Wen Li Phys. Rev. A 96, 023401 - Published 2 August 2017

DOI: 10.1103/PhysRevA.96.023401 


\title{
Coulomb-Repulsion-Assisted Double Ionization from Doubly Excited States of Argon
}

\author{
Qing Liao ${ }^{1,2}$, Alexander H. Winney ${ }^{1}$, Suk Kyoung Lee ${ }^{1}$, Yun Fei Lin ${ }^{1}$, PradipAdhikari ${ }^{1}$, and Wen \\ $\mathrm{Li}^{1, *}$ \\ wli@,chem.wayne.edu \\ ${ }^{1}$ Department of Chemistry, Wayne State University, Detroit, Michigan 48202, USA \\ ${ }^{2}$ Laboratory of Optical Information Technology, Wuhan Institute of Technology, Wuhan 430205, \\ China
}

We report a combined experimental and theoretical study to elucidate nonsequential double ionization dynamics of argon atoms at laser intensities near and below the recollision induced ionization threshold. Three-dimensional momentum measurements of two electrons arising from strong field nonsequential double ionization are achieved with a novel electron-electron-ion coincidence apparatus, showing laser intensity dependent Coulomb repulsion effect between the two outgoing electrons. Furthermore, a previously predicted feature of double ionization from doubly excited states is confirmed in the distributions of sum of two-electron momenta. A classical ensemble simulation suggests that Coulomb-repulsion-assisted double ionization from doubly excited states is at play at low laser intensity. This mechanism can explain the dependence of Coulomb repulsion effect on the laser intensity, as well as the transition from side-by-side to back-to-back dominant emission along the laser polarization direction.

PACS numbers: 32.80.Rm, 31.90.+s, 32.80.Fb

\section{Introduction}

Strong field nonsequential double ionization (NSDI) of argon atoms has attracted much interest in recent years due to its complex mechanisms and the involvement of laser-induced electron correlation [1]. When the kinetic energy of the recolliding electron is not high enough to kick out the bound electron through an (e, 2e)-like direct impact ionization (DII) process [2], the bound electron can be ejected via a recollision-induced excitation with subsequent ionization (RESI) mechanism [3, 4]. It was expected that the second ejected electron is independent of the recolliding electron after being populated to singly excited states (SES) by recollision in RESI mechanism and hence no correlation effect is expected $[4,5]$. However, this is inconsistent with the later experimental findings of dominant side-by-side emission near the recollision threshold intensities (1.5 and $0.9 \times 10^{14} \mathrm{~W} / \mathrm{cm}^{2}$, respectively) [6]. It has been observed when lowering the intensity to $(4-7) \times 10^{13} \mathrm{~W} / \mathrm{cm}^{2}$, the parallel momentum correlation switches from side-by-side (along same direction) to back-to-back (into opposite sides) dominant emission [7]. A more striking difference between the studies at high and low intensities was the complete loss of electron repulsion in the plane perpendicular to the laser polarization direction regardless of parallel momentum correlation when reducing the intensity from $1.9 \times 10^{14} \mathrm{~W} / \mathrm{cm}^{2}$ [8] to (4$7) \times 10^{13} \mathrm{~W} / \mathrm{cm}^{2}$ [7]. It was suggested that RESI mechanism involving doubly excited states (DES) dominates the NSDI dynamics below the threshold [9-14]. However, other supporting evidence of DES-RESI mechanism has not been observed experimentally so far. A semiclassical simulation [12] suggested that the distributions of sum of momenta of electrons can be used to distinguish DES-RESI from SES-RESI and DII mechanisms. The details on what leads to the transition from dominant side-by-side to dominant back-to-back emission and the disappearance 
of final state Coulomb repulsion effect in argon NSDI at low intensities are still not fully understood. One reason might be that fully differential measurements of electron spectra of argon NSDI have been scarce due to the difficulty of detecting two electrons with high efficiency. It is worth noting that argon NSDI has also been studied with few cycle laser pulses in which SES-RESI was found to play a major role $[5,15]$.

Here we report fully differential momentum measurements of NSDI of argon by $30 \mathrm{fs}, 800 \mathrm{~nm}$ laser pulse at intensities of $\sim 1.2$ and $1.5 \times 10^{14} \mathrm{~W} / \mathrm{cm}^{2}$. The intensities are chosen in such a way that the maximum kinetic energies of the recolliding electrons $(\sim 23$ and $28 \mathrm{eV}$, respectively) are below and at the field-free ionization potential of argon ion $(27.6 \mathrm{eV})$ but above the maximally suppressed ionization potential in the field $(\sim 9 \mathrm{eV})$. We observed clear Coulomb repulsion effect for side-by-side emission and its strength reduces with lower laser intensity. Based on current and previous experimental findings, we conclude that Coulomb repulsion effect as reflected in the perpendicular momentum correlation diminishes gradually with decreasing laser intensity. In addition, we find the distributions of sum of momenta (both parallel and perpendicular to the laser polarization) peak around zero only for electrons with low total energies, confirming the predicted feature of DES-RESI pathway [12]. Furthermore, our classical ensemble calculation suggests that Coulomb repulsion energy can be released to assist field double ionization from doubly excited states at low laser intensities or in final continuum states at high laser intensities. At low laser intensities, this leads to a sequential release of electron from the doubly excited states.

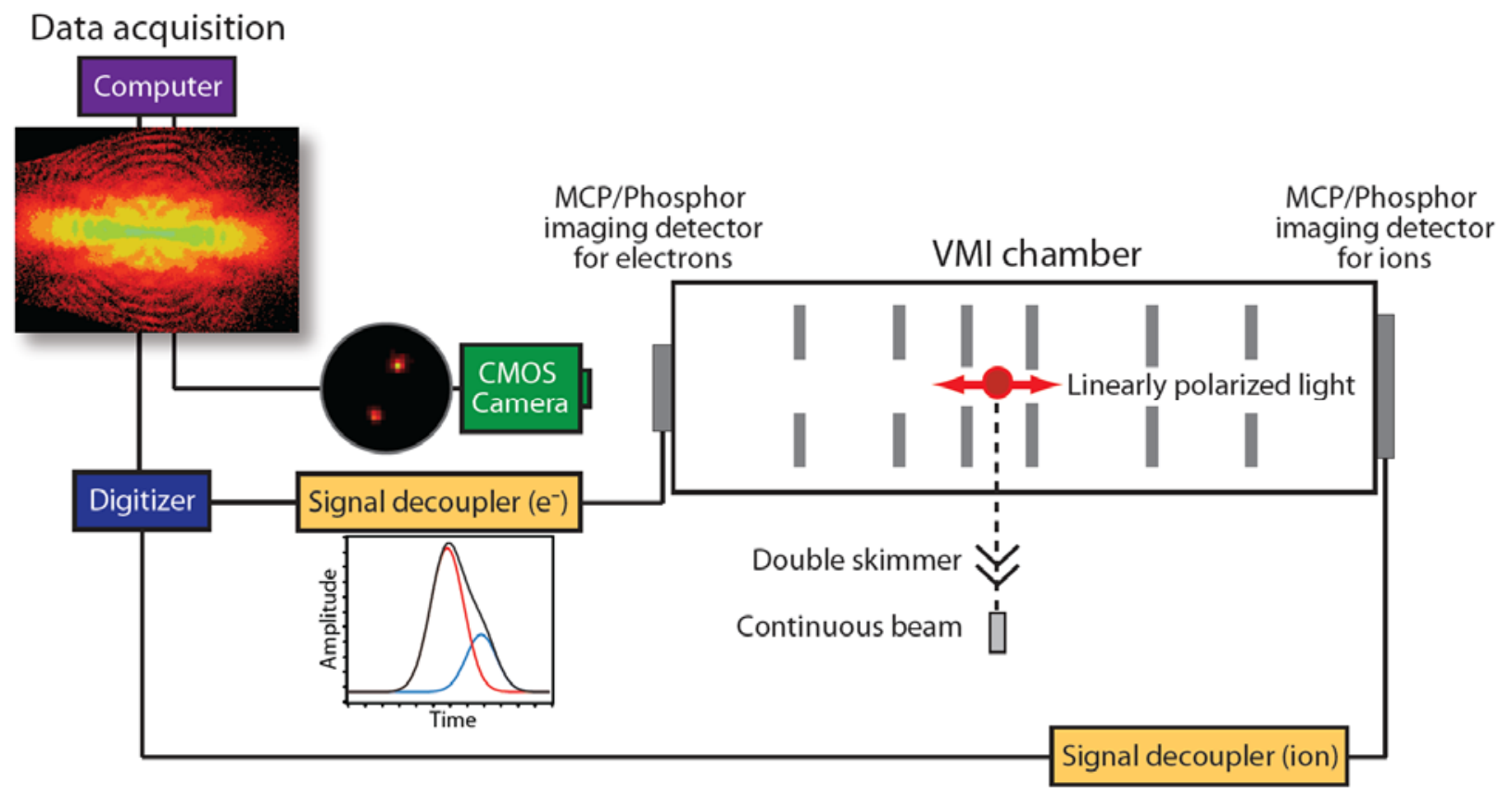

FIG. 1. (color online). The experimental setup for 3D coincidence measurements of two electrons arising from Ar NSDI. The electron imaging system is capable of electron-electron detection with a zero deadtime. The red dot and arrows in the middle of the apparatus indicates the laser beam and its polarization direction (along the TOF axis). 


\section{Experimental methods and results}

The experiment was carried out in a velocity map imaging (VMI) coincidence measurement apparatus (Fig. 1). This apparatus features a 6-electrode ion/electron optics that can velocity focus both ions and electrons to improve momentum resolution. The laser system was a 16 $\mathrm{mJ} /$ pulse, $\sim 30 \mathrm{fs}$, one $\mathrm{kHz}$ Ti:Sapphire (center wavelength $800 \mathrm{~nm}$ ) amplification system (KMLabs, Red Dragon) and the power used here was only $\sim 5 \mu \mathrm{J}$. The laser beam was focused onto the molecular beam by a spherical mirror (focal length $=10 \mathrm{~cm}$ ) mounted on a kinematic mirror mount in the vacuum. The laser polarization is along the time-of-flight TOF axis (z axis). Argon gas entered the vacuum chamber through a 20-micron diameter aperture and the beam was double skimmed before entering the main chamber. The atomic beam propagation direction was orthogonal to the TOF axis. The produced ions and electrons were then directed in opposite directions by an inhomogeneous electric field and impacted on two different $\mathrm{MCP} / \mathrm{phosphor}$ screen imagers at the end of their respective TOF regions. The electric field strength in the interaction region was $\sim 20 \mathrm{~V} / \mathrm{cm}$.

We directly measured the 3D momenta of both electrons in coincidence with argon dications with a camera-based 3D imaging system, which features a zero dead-time capability [16-18]. Because there is no dead-time in detecting two electrons, the overall detection efficiency is mainly determined by the electron detection efficiency of the microchannel plates (MCP), which is typically $50 \%$ in our set-up. Therefore, with this $3 \mathrm{D}$ imaging system, all $3 \mathrm{D}$ momenta of two electrons can be measured while the count rate can be as high as $50 \%$ of single electron detection.

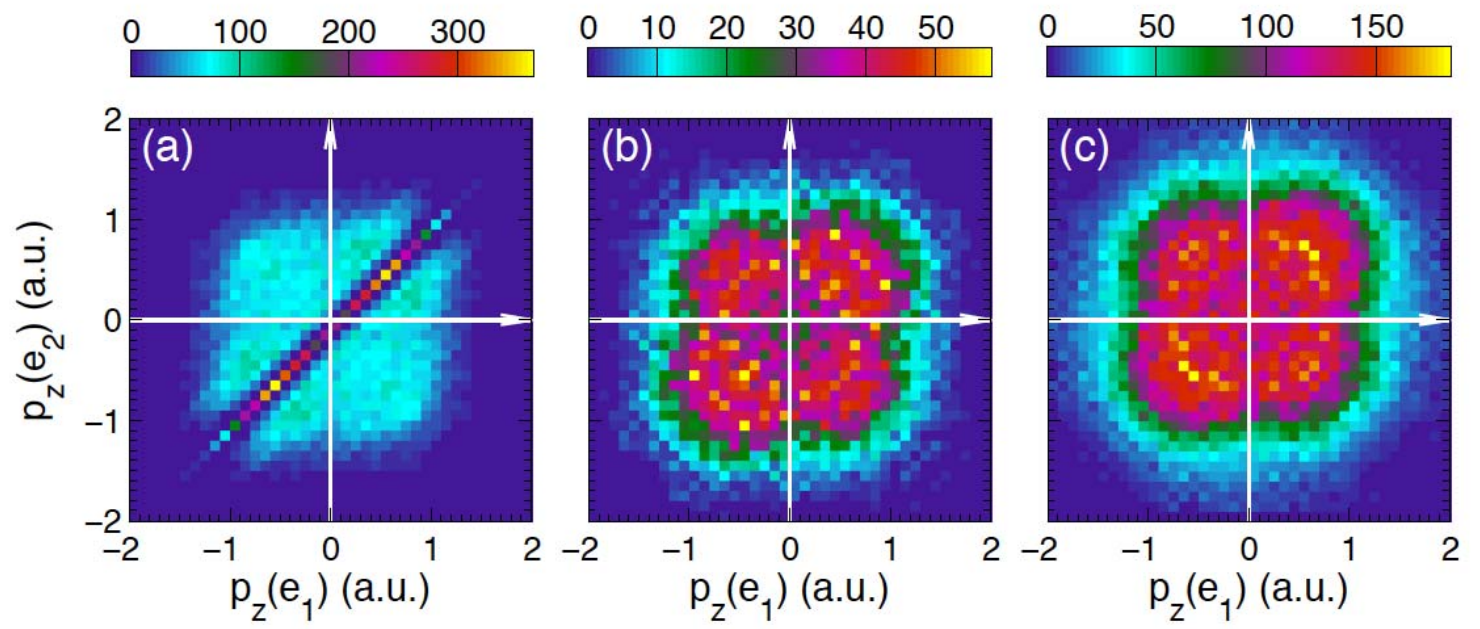

FIG. 2. (color online). Correlated momentum distributions for double ionization of Ar at $1.5 \times 10^{14} \mathrm{~W} / \mathrm{cm}^{2}$ measured with (a) a zero dead-time 3D imaging system and (c) conventional COLTRIMS approach, respectively. (b) is the same as (a) but with momentum correction, see text. $p_{z}\left(e_{1}\right)$ and $p_{z}\left(e_{2}\right)$ are the momenta of electron 1 and electron 2 parallel to the laser polarization direction, respectively.

Previously, we have shown this apparatus can measure electron momentum correlation in NSDI of benzene [19] by detecting two electrons, with which a cold target is difficult to achieve and thus the conventional COLTRIMS approach (measuring electron-dication double coincidence and using momentum conservation to infer the momentum of the second electron) becomes 
unreliable. However, even though the method is highly efficient in detecting two electrons, there was one issue associated with it: if the TOFs of two electrons are extremely close $(<1 \mathrm{~ns})$, the employed computer algorithm will assign the same TOF to both electrons and this introduces some uncertainty in the momentum measurement (see in Figure 2 (a), the diagonal line and surrounding blank area). These uncertainties do not qualitatively change the results of NSDI studies. However, with atomic targets such as argon, by measuring the dication momentum and using momentum conservation, it is possible to remove such artifact along the TOF axis and also improve the accuracy of the momenta perpendicular to the TOF axis of both electrons. In this work, we achieved a momentum resolution of $<0.2$ a.u. for ions and this allows momentum conservation to be used for events with very similar TOFs by assuming the measured TOF belongs to the electron which produces more secondary electrons in MCP. Furthermore, only those events with calculated second electron momentum fall in the range of -1.5 a.u. and 1.5 a.u. were considered valid events. The result of momentum correlation along the laser polarization is shown in Figure 2 (b) while Figure 2(c) was derived with the conventional COLTRIMS approach using double coincidence. The agreement between these is quite good and shows the camera-based 3D system can indeed achieve true zero-dead time measurement of the 3D momenta of two electrons. In this experiment, the overall count rate for electrons was about 0.11 per laser shot while that of ions was 0.06 per laser shot. We estimated the false coincidence rate to be less than $20 \%$.
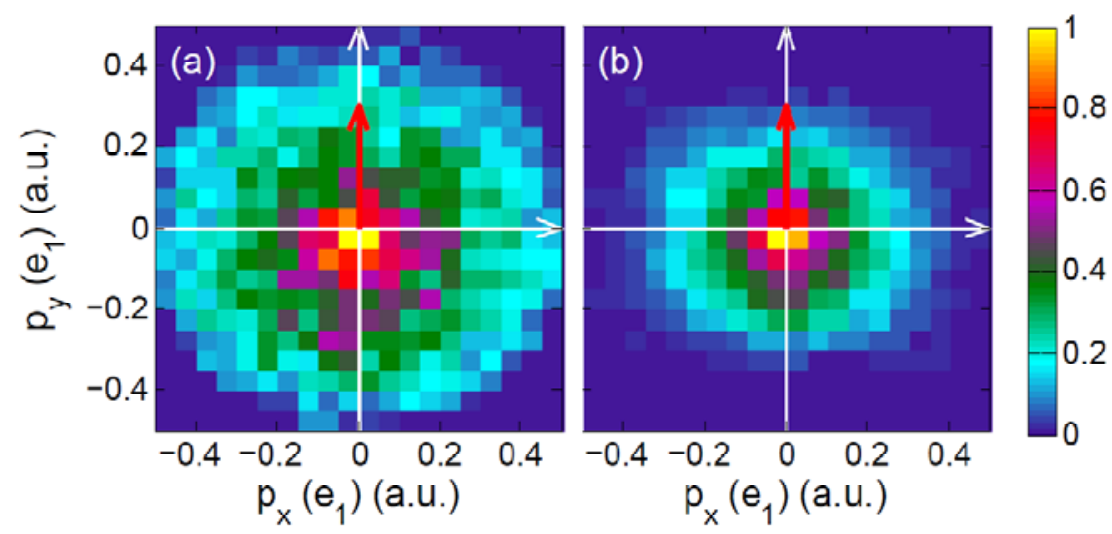

FIG.3. (color online). Two-dimensional perpendicular momentum distribution of electron 1 while the $y$ momentum component of electron 2 is restricted to be positive as indicated by the red arrow for side-byside double ionization events. The laser intensities are (a) $1.5 \times 10^{14} \mathrm{~W} / \mathrm{cm}^{2}$ and (b) $1.2 \times 10^{14} \mathrm{~W} / \mathrm{cm}^{2}$, respectively.

From figures 2(a) and 2(b), we can see there are both side-by-side (events in the quadrants 1 and 3 ) and back-to-back (events in the quadrants 2 and 4) emissions, although side-by-side emission has $\sim 17 \%$ more yield than back-to-back events. Fig. 3(a) shows the momentum distribution of one electron in the plane perpendicular to the polarization direction for side-by-side events from Fig. 2(b). Evident final state Coulomb repulsion effect is found when the second electron's momentum is defined along positive y axis, as indicated by the red arrow (note y is arbitrary and not associated with any certain direction in laboratory frame). The events with negative $y$ momentum component of the first electron are $\sim 37 \%$ more than those with positive y momentum component. We find the repulsion effect is weaker than that at $1.9 \times 10^{14} \mathrm{~W} / \mathrm{cm}^{2}$ (see Fig. 1(a) in Ref. [8]). It is also weaker than the repulsion effect for neon double ionization at the recollision 
threshold intensity of $1.9 \times 10^{14} \mathrm{~W} / \mathrm{cm}^{2}$ (see Fig. 1(b) in Ref.[20]). When the laser intensity decreases to $1.2 \times 10^{14} \mathrm{~W} / \mathrm{cm}^{2}$, the repulsion effect becomes further weaker, as shown in Fig. 3(b) from side-by-side events, measured without momentum correction. We find $\sim 31 \%$ more events in negative $\mathrm{y}$ momentum region than in positive $\mathrm{y}$ momentum region. Note that our measurements with and without momentum correction do not affect the perpendicular momentum distribution. This is confirmed by comparing the perpendicular momentum distributions for events from Figs. 2(a) and 2(b).
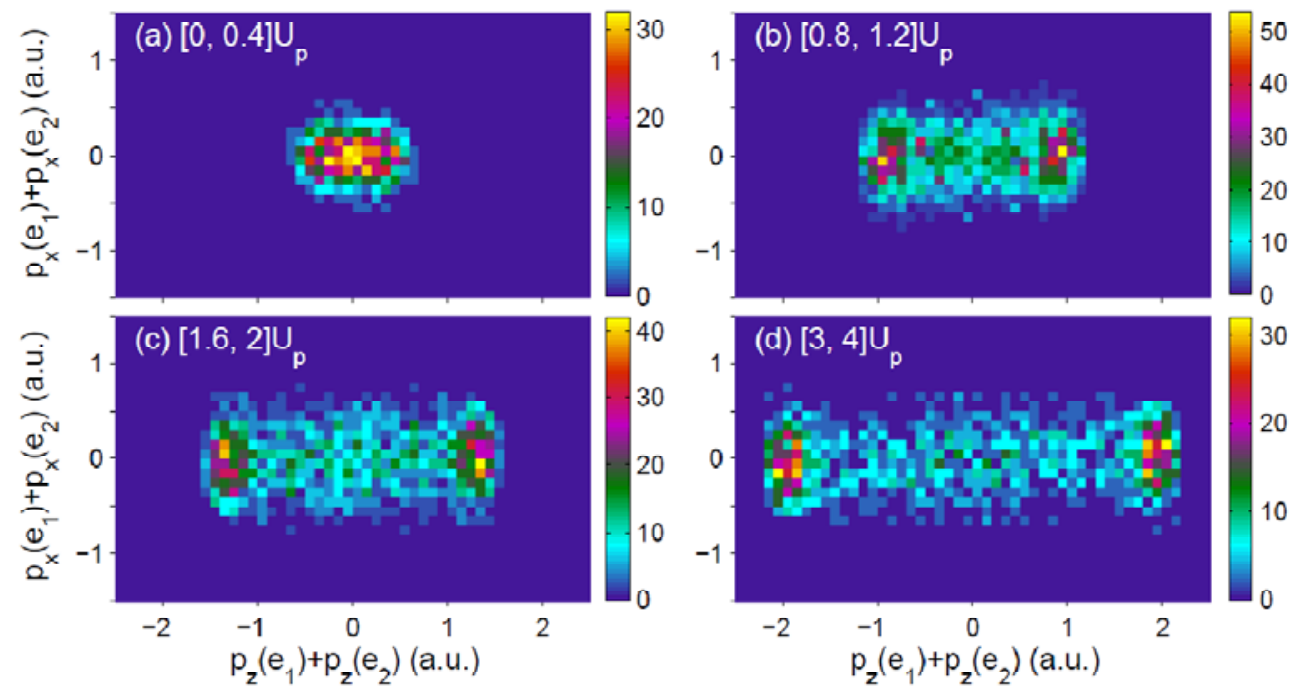

FIG.4. (color online). Sum of momenta distributions in directions parallel and perpendicular to the laser polarization for double ionization at $1.5 \times 10^{14} \mathrm{~W} / \mathrm{cm}^{2}$ with total energy ranges in (a) $[0,0.4] \mathrm{U}_{\mathrm{p}}$, (b) $[0.8$, $1.2] \mathrm{U}_{\mathrm{p}}$, (c) $[1.6,2] \mathrm{U}_{\mathrm{p}}$, and (d) $[3,4] \mathrm{U}_{\mathrm{p}}$, where $\mathrm{U}_{\mathrm{p}}$ is the ponderomotive energy.

The final state Coulomb repulsion effect suggests that the second electron is emitted in close proximity of the first one temporally after DESs are populated by recollision [20]. However, even though double ionization mechanism involving DESs has been proposed previously, no supporting evidence other than momentum correlation has been observed. A semiclassical simulation [12] pointed out that for electrons with low total energy, the sums of momenta in all three directions are close to zero for DES-RESI processes. This is different from those resulted from DII and SES-RESI mechanisms, which show two sharp peaks in the direction parallel to the laser polarization (see Fig. 4 in Ref. [12]). This is well understood because for DES-RESI mechanism the momenta of both electrons tend to be zero (ionization at the peak of the laser electric field), while in the other mechanisms at least one electron is freed at recollision and thus gains a high momentum. Our experimental result does exhibit the feature of DES-RESI mechanism, as shown in Fig. 4(a), and thus implies that DES-RESI mechanism is dominant in the low total energy range. This is not the case for higher total energy events due to less important contribution of DES-RESI [see Figs. 4(b)-4(c)].

\section{Theoretical methods and comparisons with experimental results}

It is expected that DES-RESI mechanism becomes dominant at very low laser intensities. Does it relate to the disappearance of Coulomb repulsion effect and the dominant back-to-back 
emissionat $(4-7) \times 10^{13} \mathrm{~W} / \mathrm{cm}^{2}$ [7]? To answer this question, we implement a fully classical ensemble simulation. The validation of employing classical calculations in the study of NSDI has been provided in numerous previous studies [21, 22], in which such calculations have provided qualitative physical insight. In the current model (for details see Refs. [22-24]), electron-nuclear and electron-electron interactions are represented by 3D soft-Coulomb potentials:

$$
V\left(\vec{r}_{1}, \vec{r}_{2}\right)=-2 / \sqrt{\left|\vec{r}_{1}\right|^{2}+a^{2}}-2 / \sqrt{\left|\vec{r}_{2}\right|^{2}+a^{2}}+1 / \sqrt{\left|\vec{r}_{1}-\vec{r}_{2}\right|^{2}+b^{2}} \text { (in atomic units). }
$$

$\vec{r}_{1}$ and $\vec{r}_{2}$ are the coordinates of the two electrons, respectively. $a$ and $b$ are the soft-core Coulomb parameters.

$$
E_{t o t}=\left(\left|\vec{p}_{1}\right|^{2}+\left|\vec{p}_{2}\right|^{2}\right) / 2+V\left(\vec{r}_{1}, \vec{r}_{2}\right)
$$

is the total energy. $\vec{p}_{1}$ and $\vec{p}_{2}$ are the momenta of the two electrons, respectively. $b$ is set to 0.05 to support the strong electron-electron interaction. Here, the total energy is set as the ground state energy of argon: -1.59 a.u. (the sum of the first and second ionization potentials: 0.58 a.u. and 1.01 a.u.). As a consequence, $a$ is generally set in the range from 1.25 to 1.6 to avoid autoionization and to keep the total potential energy $V\left(\vec{r}_{1}, \vec{r}_{2}\right)$ less than the ground state energy. We first assigned random positions and momenta to the two electrons satisfying equation (2) as a starting point and then let them move under Newtonian equations of motion. The total energy remains the same during the evolution without laser fields. Finally, we recorded the corresponding trajectory and obtained the positions and momenta of the trajectory at every time interval of 0.25 a.u. as the initial ensemble with a size of 1 million. To obtain reasonable initial ensemble (see Figure 5), the best value of $a$ should be set in the range from 1.4 to 1.5. It was set as 1.5 in previous studies [25].
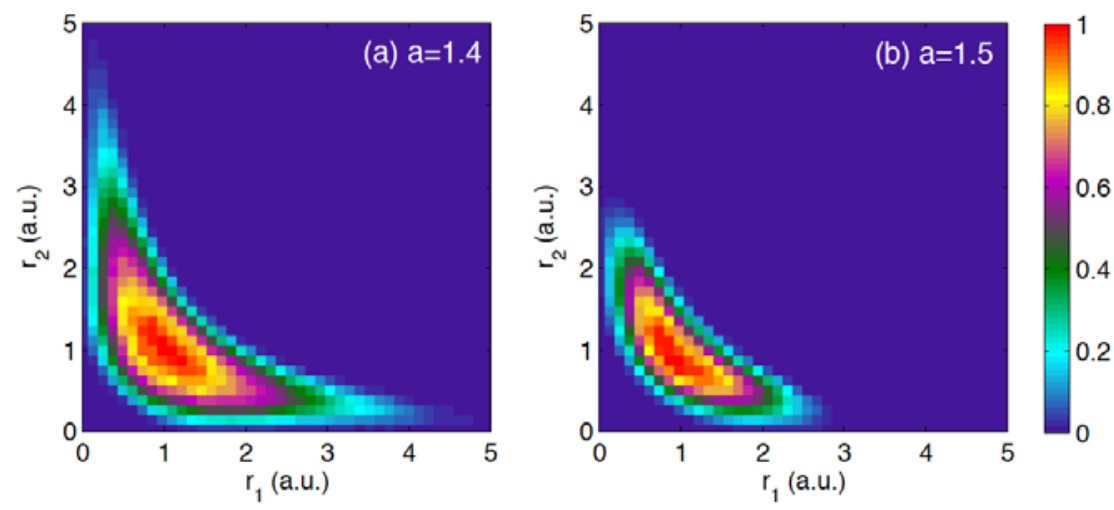

FIG. 5. (color online) Normalized initial ensemble spatial distribution as functions of the distances of the two electrons from the nucleus, $r_{1}$ and $r_{2}$ respectively. The soft-core Coulomb parameter $a=1.4$ (a) and $a=1.5(\mathrm{~b})$.

Starting with the initial ensemble, the trajectories of all individual electron pairs are recorded under a laser electric field of a total duration of 16 optical cycles (switched on and off linearly over 3 optical cycles, respectively). After the laser pulse is over, the system is propagated for additional 16 optical cycles to ensure there is no interaction between the two escaped electrons. Double ionization events are defined for trajectories with positive energies for both electrons at 
the final time. Fig. 6 shows the calculated momentum distributions along the laser polarization direction for $a=1.4$ and 1.5 at a laser intensity of $1.5 \times 10^{14} \mathrm{~W} / \mathrm{cm}^{2}$. By comparing with experimental results, $a$ is adjusted to 1.4 to best reproduce the correlated momentum distribution, slightly different from the usually employed value in Ref. [25]. In the following texts, we will use $a=1.4$ to calculate NSDI at different intensities. Our model with these parameters is able to reproduce qualitatively the trend of momentum correlation at different laser intensities observed in previous experiments. It should be noted that a slight change of $a$ can affect the respective contributions of different NSDI mechanisms quantitatively, but does not change our main conclusions.
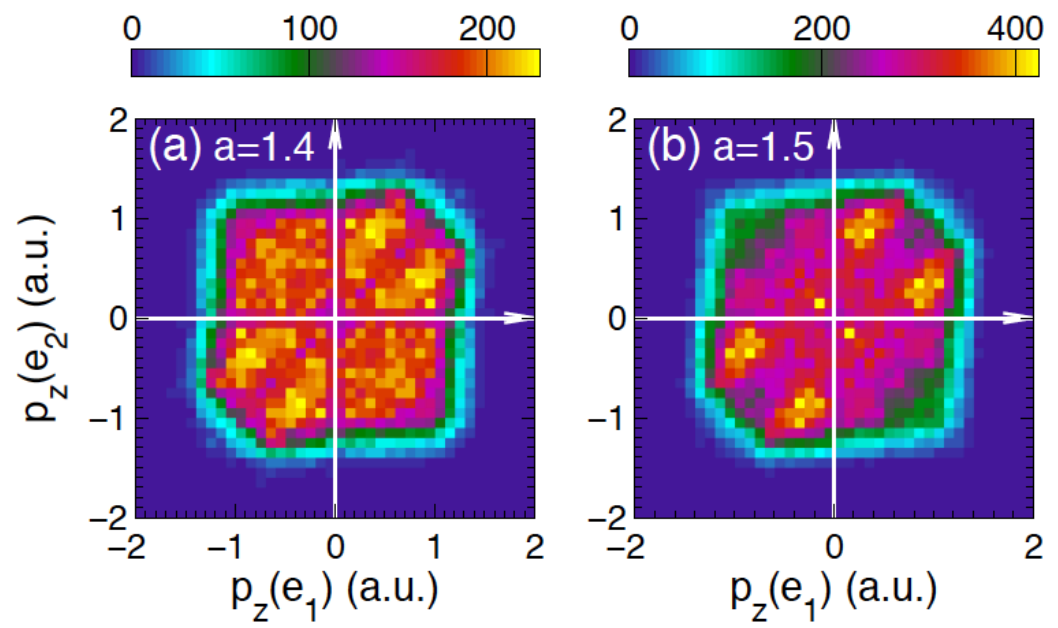

FIG. 6. (color online) Calculated momentum distributions along the laser polarization direction at a laser intensity of $1.5 \times 10^{14} \mathrm{~W} / \mathrm{cm}^{2}$. The soft-core Coulomb parameter $a=1.4$ (a) and $a=1.5$ (b).

From Fig. 6 (a), at $1.5 \times 10^{14} \mathrm{~W} / \mathrm{cm}^{2}$, side-by-side events have $\sim 13 \%$ more yield than back-toback events, which is comparable with the $17 \%$ difference measured from experiments. At $1.2 \times 10^{14} \mathrm{~W} / \mathrm{cm}^{2}$ (momentum data not shown), the theoretical and experimental values are $2 \%$ and $10 \%$, respectively.

In Fig. 7, we showed the calculated intensity dependent Coulomb repulsion effect in the perpendicular direction (see Fig. 3 for experimental results). The events with negative y momentum component of the first electron are $\sim 42 \%$ (37\% from the experiment) and $\sim 38 \%$ (31\% from the experiment) more than those with positive y momentum component for $1.5 \times 10^{14} \mathrm{~W} / \mathrm{cm}^{2}$ and $1.2 \times 10^{14} \mathrm{~W} / \mathrm{cm}^{2}$, respectively. Qualitatively, the measured angular correlations trend at different laser intensities is in good agreement with the calculated one, even though the measured absolute momentum difference is larger.
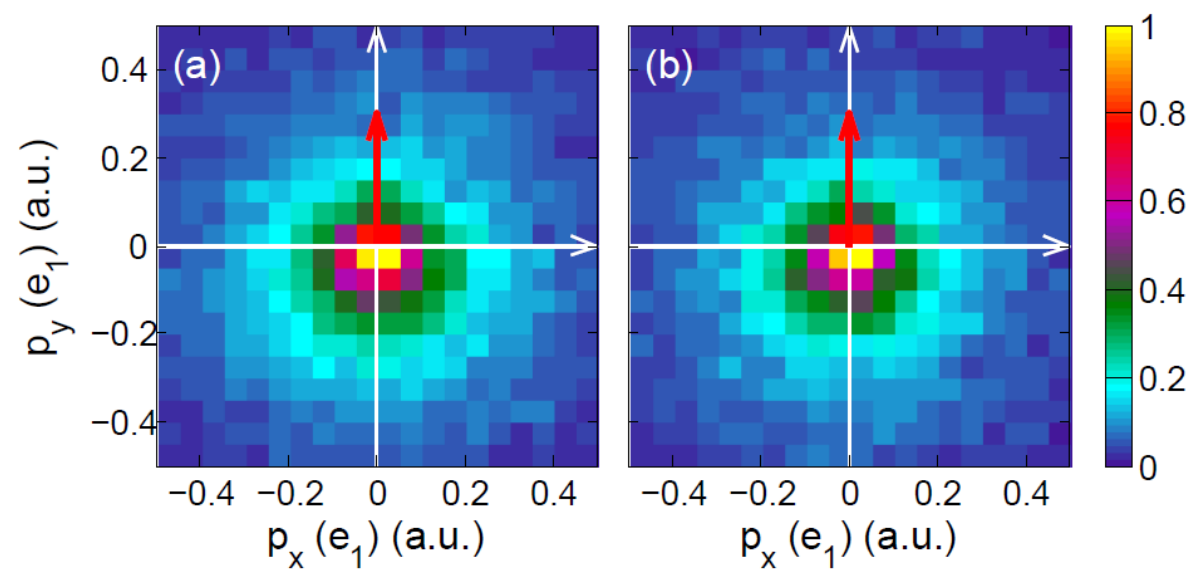
FIG. 7. (color online). Same as Fig. 3 but from the calculations. The laser intensities are (a) $1.5 \times 10^{14}$ $\mathrm{W} / \mathrm{cm}^{2}$ and (b) $1.2 \times 10^{14} \mathrm{~W} / \mathrm{cm}^{2}$, respectively.

Fig. 8 shows the calculated distributions of sum of momenta along the directions parallel and perpendicular to the laser polarization (see Fig. 4 for experimental results). Similar as previous calculation results [12], for events with a low total energy, the momentum sum in both directions approach zero, which was attributed to DESI-RESI dynamics.
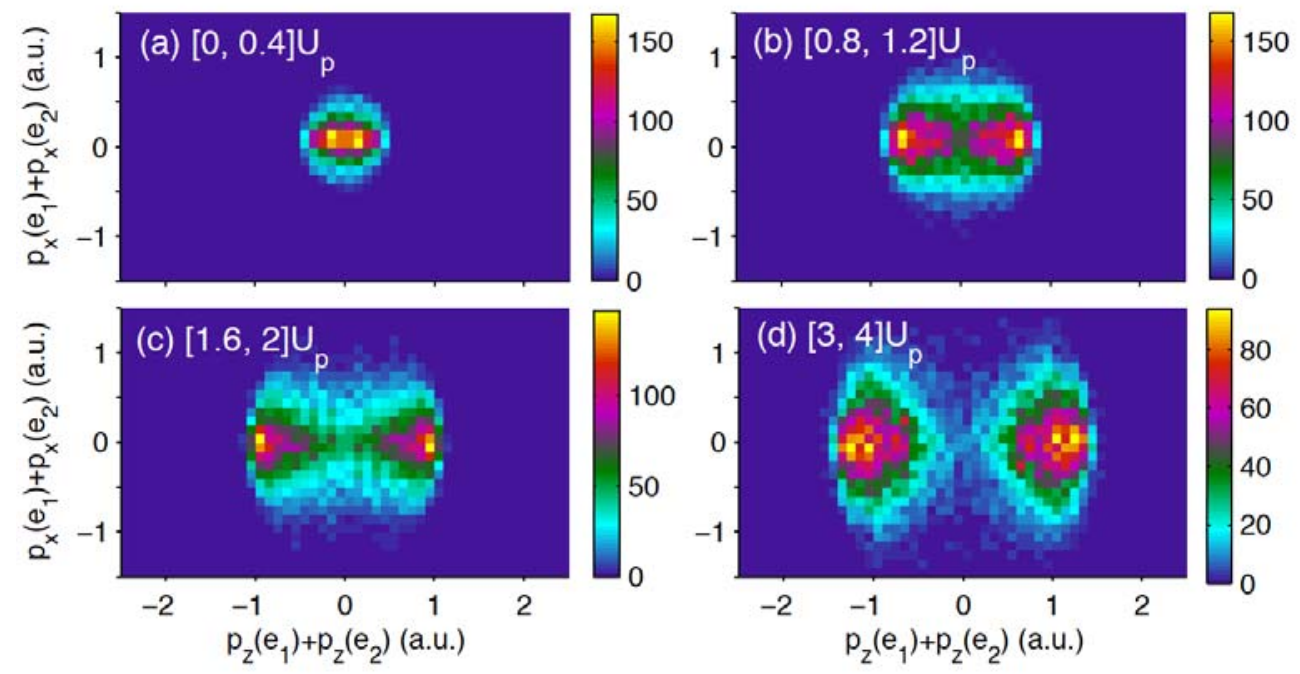

FIG. 8. (color online). Same as Fig. 4 but from the calculations.

\section{Coulomb-repulsion-assisted double ionization}

Now that we have shown the calculated results are in agreement with those from experiments, further insight on NSDI dynamics can be extracted from the calculation due to its classical nature. We achieved this by selecting out DES and SES events. First we back-tracked all double ionization trajectories and identify a recollision trajectory according to the following criterion: the first electron moves far away from the ion ( $>8$ a.u.) and then returns to the ion while the other one keeps in the vicinity of the nucleus (distance $<3$ a.u.). If the two electrons can reach a nearest distance less than 5 a.u. and at this moment both electron-nucleus distances are also less than 5 a.u., this moment is considered a recollision moment and the trajectory a recollision one. With this procedure, at least $96 \%$ of trajectories that lead to final double ionization are identified as recollision trajectories. For these recollision trajectories, we then calculated the total energy of the two electrons at the last recollision moment (recollision may happen many times, especially at low laser intensities). If the total energy is negative, the event is considered a RESI event, and otherwise a DII event. For RESI events, if the energies of both electrons are negative at the last recollision moment, the trajectory is identified as a DES-RESI event, while if the energy of one electron is negative and that of the other is positive, the trajectory is identified as a SES-RESI 
event. Here the two electrons share the Coulomb repulsion energy equally when we calculate the individual energy. DES events are excluded from the initial ensemble by confirming the energies of all DESs having energies above the ground state energy of $\operatorname{Ar}^{+}(-1.01$ a.u.).

Figure 9(a) shows the dependence of DES events and SES events on the laser intensity. As the intensity decreases, DES contribution increases while SES contribution drops. At low intensities, DES events are clearly dominant, in agreement with previous calculations $[11,14]$. However, at rather high intensity $2 \times 10^{14} \mathrm{~W} / \mathrm{cm}^{2}$, DES-RESI still plays an important role. This is somewhat surprising because it has been suggested that DES-RESI would only play a role at much lower intensity $\left(<1.2 \times 10^{14} \mathrm{~W} / \mathrm{cm}^{2}\right)$ [14]. Our experimental results at $1.5 \times 10^{14} \mathrm{~W} / \mathrm{cm}^{2}$ confirms there is indeed significant contribution of DES-RESI. It should be noted at higher intensities $\left(>4 \times 10^{14}\right.$ $\mathrm{W} / \mathrm{cm}^{2}$ ), DII mechanism begins to take over.
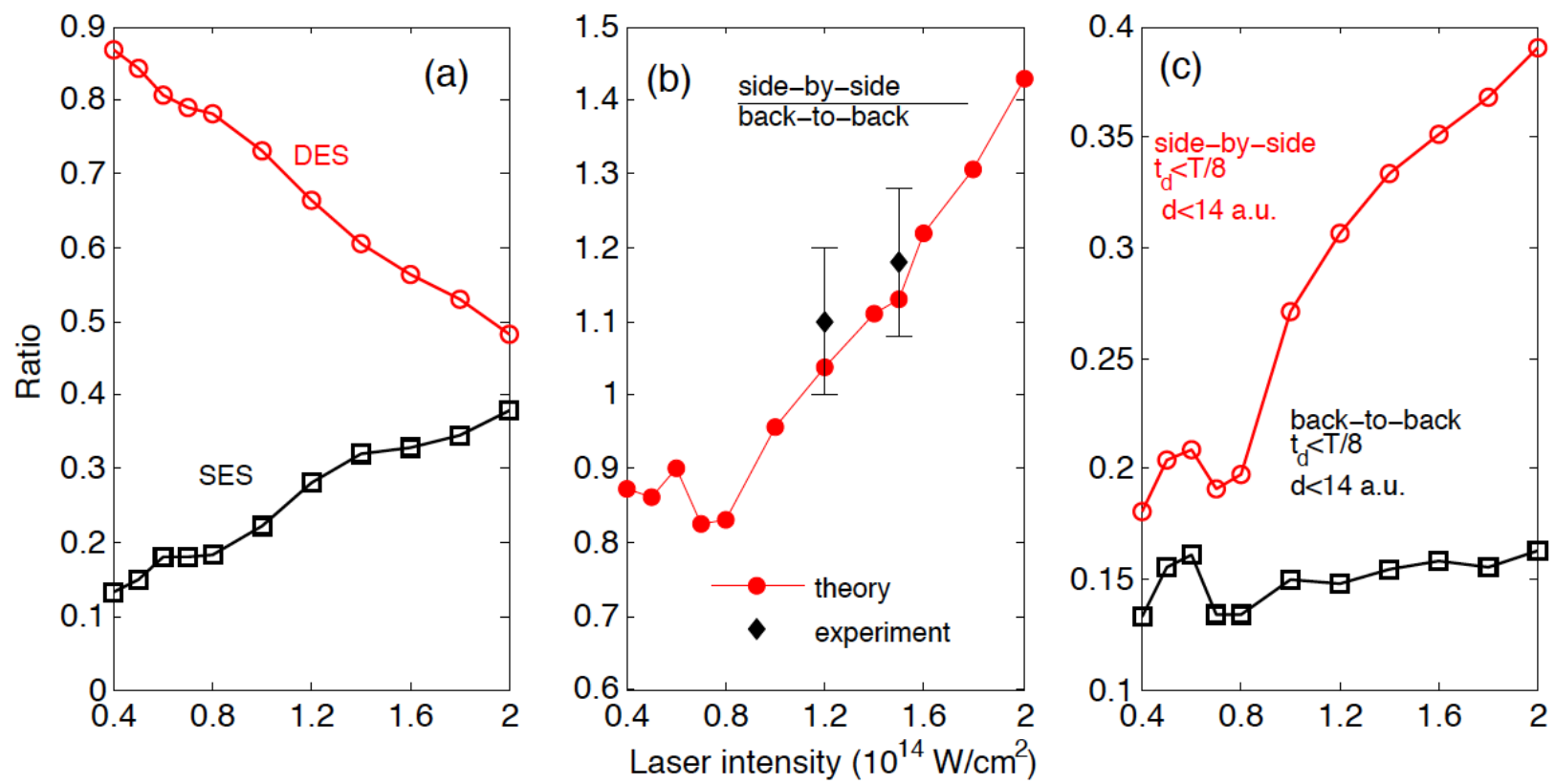

FIG. 9. (color online) (a) Ratios of DES and SES events to the total double ionization events as a function of laser intensity. (b) Within DES events, the ratios between side-by-side and back-to-back events as a function of laser intensity (red solid circles). The ratios from experiments at $1.2 \times 10^{14} \mathrm{~W} / \mathrm{cm}^{2}$ and $1.5 \times 10^{14} \mathrm{~W} / \mathrm{cm}^{2}$ are also shown (black solid diamonds). Note the experimental results include contributions from different mechanisms (DES-RESI, SES-RESI and DII) while the trend is dominated by DES-RESI. (c) The contribution of side-by-side DES events with ionization time delays $t_{d}<T / 8$ and relative distances between the two electrons at the second ionization time $d<14$ a.u. (open red circles) to the total side-by-side DES events as a function of laser intensity. Open black squares: the same but for back-to-back DES events.

Within DES-RESI mechanism, the contribution of side-by-side events decreases while that of back-to-back events increases until the laser intensity is reduced to $0.8 \times 10^{14} \mathrm{~W} / \mathrm{cm}^{2}$, and then keep almost unchanged, see Fig. 9(b). Further analysis on the relative ionization time delay $t_{d}$ between the two electrons and their relative distance $d$ at the second ionization time shows that the contribution of side-by-side events with $t_{d}<T / 8$ ( $\mathrm{T}$ is the laser period) and $d<14 \mathrm{a}$. $\mathrm{u}$. to the 
total side-by-side events drops quickly with reducing intensity, see Fig. 9(c). Here we define the ionization time as the first moment when one electron is at least 6 a.u. away from the ion after the last recollision. Note that $t_{d}<T / 8$ and $d<14$ a.u. mean that the two electrons are emitted closely temporally and spatially and these events should show final state Coulomb repulsion effect. For back-to-back events, the contributions from small $t_{d}$ and small $d$ are always very low, implying no Coulomb repulsion effect even for high intensities. Based on these analysis, we can rationalize the experimental findings at different laser intensities in the following way (see Fig. 10): if Coulomb repulsion energy is released in perpendicular direction as in the case of side-byside events [step (2) in Fig. 10(a)], the actual ionization potential is effectively higher than those without Coulomb repulsion effect; this makes these events less likely to happen at low laser intensities. Instead, Coulomb repulsion energy prefers release in the laser polarization direction to assist field ionization for low intensities [step (1) in Fig. 10(b)]. At lower intensities, more repulsion energy is released in this direction, resulting in a weaker repulsion effect in perpendicular direction. The repulsion energy release process also results in longer ionization time delays. Since both electrons tend to be ionized near the field maximum (the crossing of the vector potential) and their final momenta are dominated by the vector potentials at the instant of ionization, they favor back-to-back emission. This explains the intensity-dependent Coulomb repulsion effect and the observed dominant back-to-back emission parallel to the laser polarization at much lower intensities. This mechanism involves a sequential release of the electrons from the doubly excited states and is consistent with a previous semiclassical calculation at very low laser intensity $\left(7 \times 10^{13} \mathrm{~W} / \mathrm{cm}^{2}\right)[14]$.

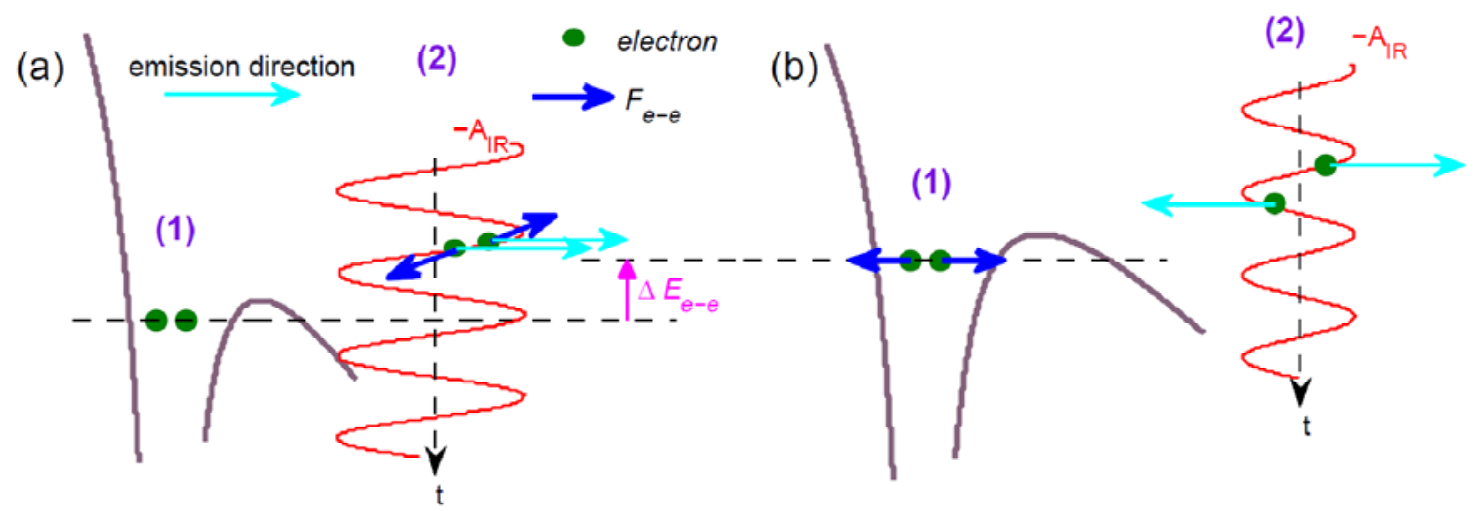

FIG. 10. (color online) Schematic of double ionization from doubly excited states with Coulomb energy $\Delta E_{e-e}$ release after tunneling ionization for a strong laser field (a) and assisted tunneling ionization process for a weak laser field (b). $F_{e-e}$ is the Coulomb repulsion force between the two electrons and $A_{I R}$ the vector potential of the laser field. (1) and (2) indicate stages before and after tunneling ionization, respectively. The energy difference $\Delta E_{e-e}$ between (a) and (b) cases is the reason why DES-RESI dynamics behaves differently at different laser intensities.

\section{Conclusions}

In summary, with a 3D electron-electron-ion coincidence detection system, we found that in Ar NSDI, the final state Coulomb repulsion effect weakens as the laser intensity decreases near the recollision threshold as the contribution of DES-RESI become more significant. We also 
demonstrated from both experiment and simulations the signature feature of DES-RESI mechanism, which manifests in the vanishing sum of momenta for electrons with low total energies. Our classical simulations further suggest that after recollision-induced double excitation, electron-electron repulsion energy tends to be released in the laser field direction to assist field double ionization at weak laser fields, leading to less repulsion energy released in final states and therefore back-to-back emission becomes more favorable.

We would like to acknowledge the US Army Research Laboratory and the US Army Research Office for financial support (Award numbers: W911NF-12-1-0598 and W911NF-14-1-0473). W. L. is partially supported by the Sloan Foundation as an Alfred P. Sloan Research Fellow.

[1] W. Becker, X. Liu, P. J. Ho, et al., Rev. Mod. Phys. 84, 1011 (2012).

[2] A. Rudenko, V. L. B. de Jesus, T. Ergler, et al., Phys. Rev. Lett. 99, 263003 (2007).

[3] T. Weber, H. Giessen, M. Weckenbrock, et al., Nature 405, 658 (2000).

[4] B. Feuerstein, R. Moshammer, D. Fischer, et al., Phys. Rev. Lett. 87, 043003 (2001).

[5] B. Bergues, M. Kübel, N. G. Johnson, et al., Nat Commun 3, 813 (2012).

[6] E. Eremina, X. Liu, H. Rottke, et al., J. Phys. B-At. Mol. Opt. Phys. 36, 3269 (2003).

[7] Y. Liu, S. Tschuch, A. Rudenko, et al., Phys. Rev. Lett. 101, 053001 (2008).

[8] M. Weckenbrock, A. Becker, A. Staudte, et al., Phys. Rev. Lett. 91, 123004 (2003).

[9] T. Brabec, M. Y. Ivanov, and P. B. Corkum, Phys. Rev. A 54, R2551 (1996).

[10] R. Panfili, S. L. Haan, and J. H. Eberly, Phys. Rev. Lett. 89, 113001 (2002).

[11] Q. Liao and P. Lu, Phys. Rev. A 82, 021403 (2010).

[12] A. Emmanouilidou, Phys. Rev. A 83, 023403 (2011).

[13] Z. Chen, Y. Liang, D. H. Madison, et al., Phys. Rev. A 84, 023414 (2011).

[14] Y. Liu, L. Fu, D. Ye, et al., Phys. Rev. Lett. 112, 013003 (2014).

[15] M. Kübel, K. J. Betsch, G. K. Nora, et al., New J. Phys. 16, 033008 (2014).

[16] S. K. Lee, F. Cudry, Y. F. Lin, et al., Rev. Sci. Instrum. 85, 123303 (2014).

[17] S. K. Lee, Y. F. Lin, S. Lingenfelter, et al., J. Chem. Phys. 141, 221101 (2014).

[18] Y. F. Lin, S. K. Lee, P. Adhikari, et al., Rev. Sci. Instrum. 86, 096110 (2015).

[19] A. H. Winney, Y. F. Lin, S. K. Lee, et al., Phys. Rev. A 93, 031402 (2016).

[20] M. Weckenbrock, D. Zeidler, A. Staudte, et al., Phys. Rev. Lett. 92, 213002 (2004).

[21] P. J. Ho, R. Panfili, S. L. Haan, et al., Phys. Rev. Lett. 94, 093002 (2005).

[22] S. L. Haan, L. Breen, A. Karim, et al., Phys. Rev. Lett. 97, 103008 (2006).

[23] Q. Liao and P. Lu, Opt. Express 17, 15550 (2009).

[24] S. L. Haan, L. Breen, A. Karim, et al., Opt. Express 15, 767 (2007).

[25] X. Wang and J. H. Eberly, J. Chem. Phys. 137, 22A542 (2012). 